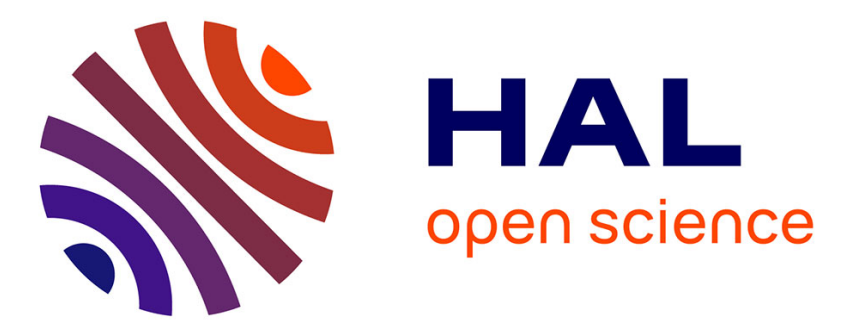

\title{
Cristaux vitreux. I : RMN large bande, thermocourants de dépolarisation et analyse enthalpique dans quelques cycloalcools
}

\author{
A. Dworkin, Alain H. Fuchs, M. Ghelfenstein, H. Szwarc
}

\section{To cite this version:}

A. Dworkin, Alain H. Fuchs, M. Ghelfenstein, H. Szwarc. Cristaux vitreux. I : RMN large bande, thermocourants de dépolarisation et analyse enthalpique dans quelques cycloalcools. Journal de Physique Lettres, 1982, 43 (1), pp.21-27. 10.1051/jphyslet:0198200430102100 . jpa-00232001

\section{HAL Id: jpa-00232001 https://hal.science/jpa-00232001}

Submitted on 1 Jan 1982

HAL is a multi-disciplinary open access archive for the deposit and dissemination of scientific research documents, whether they are published or not. The documents may come from teaching and research institutions in France or abroad, or from public or private research centers.
L'archive ouverte pluridisciplinaire HAL, est destinée au dépôt et à la diffusion de documents scientifiques de niveau recherche, publiés ou non, émanant des établissements d'enseignement et de recherche français ou étrangers, des laboratoires publics ou privés. 
Classification

Physics Abstracts

$64.70 \mathrm{E}-64.70 \mathrm{~K}-65.40-76.60 \mathrm{E}-77.30-77.40$

\title{
Cristaux vitreux. I : RMN large bande, thermocourants de dépolarisation et analyse enthalpique dans quelques cycloalcools $(*)$
}

\author{
A. Dworkin, A. H. Fuchs, M. Ghelfenstein et H. Szwarc \\ Laboratoire de Chimie Physique des Matériaux Amorphes (**) \\ Université Paris-Sud, 91405 Orsay Cedex, France \\ (Reçu le 3 juillet 1981, révisé le 30 octobre, accepté le 12 novembre 1981)
}

\begin{abstract}
Résumé. - Une série de cycloalcools a été étudiée par RMN large bande, thermocourants de dépolarisation et analyse enthalpique différentielle. Dans tous les cas, du cyclobutanol au cyclooctanol, un cristal vitreux a été observé. Le domaine de fréquences exploré $\left(10^{5}-10^{-7} \mathrm{~Hz}\right)$ permet de mettre en évidence la persistance de réorientations moléculaires en dessous de la température de transition vitreuse ainsi que leur rôle probable dans l'existence du domaine d'absorption diélectrique secondaire. L'extension de la gamme de fréquences vers $3 \times 10^{7}-10^{12} \mathrm{~Hz}$ paraît nécessaire.
\end{abstract}

\begin{abstract}
A homologous series of cycloalcohols was studied by NMR line width measurements, thermally stimulated depolarization currents and differential scanning calorimetry. In all compounds from cyclobutanol to cyclooctanol, a glassy crystal was obtained. In the investigated frequency domain $\left(10^{5}-10^{-7} \mathrm{~Hz}\right)$, it was shown that reorientational motions of the molecules still occur below the glass transition temperature and thus might be involved in the secondary dielectric relaxation process. Further investigations at higher frequencies $\left(3 \times 10^{7}-10^{12} \mathrm{~Hz}\right)$ are scheduled.
\end{abstract}

1. Introduction. - Dans leur classification empirique des transitions de phase établie pour les cristaux moléculaires sur la base de mesures thermodynamiques, Westrum et McCullough [1] avaient noté la ressemblance de certaines d'entre elles avec la transition vitreuse. Comme elles se produisaient seulement dans des cristaux obtenus par refroidissement de phases réorientationnelles, ils eurent l'intuition qu'elles étaient, d'une façon ou d'une autre, reliées au " gel " de mouvements de réorientation. Séki et al. [2] observèrent une relaxation enthalpique au-dessous d'une telle transition dans le cyclohexanol et en déduisirent qu'ils avaient affaire dans ce cas à une phase thermodynamiquement instable, qu'ils baptisèrent " cristal vitreux ».

(*) Cet article a été présenté au Congrès de la Société Française de Physique (Clermont-Ferrand, juillet 1981) dans le cadre du Colloque "Verres et Amorphes ».

This article was presented at the Meeting of the French Physical Society (Clermont-Ferrand, July 1981) at the Symposium on " Glass and Amorphous Materials ».

(**) Partie du LA 75. 
Les mesures radiocristallographiques d'Otsûbo et Sugawara [3] avaient montré l'identité des réseaux cristallins du cristal vitreux du cyclohexanol et de la phase réorientationnelle dont il est issu; Séki et al. en déduisirent que l'élément de désordre résiduel était fourni par la diversité des orientations moléculaires. Cette hypothèse a été confortée récemment par Ceccaldi et al. [4] grâce à des mesures de diffusion des rayons $\mathrm{X}$.

Cet état d'agrégation de la matière constitue un intermédiaire attrayant pour qui veut étudier l'état vitreux, puisque, par contraste avec un verre habituel où le désordre intéresse à la fois la position et l'orientation des molécules, il n'affecterait ici que les orientations moléculaires.

La transition vitreuse est un phénomène cinétique et il nous a donc semblé indispensable d'employer des méthodes expérimentales couvrant une gamme de fréquences aussi large que possible. C'est ainsi que nous avons utilisé la RMN large bande $\left(\sim 10^{5} \mathrm{~Hz}\right)$, les thermocourants de dépolarisation $\left(10^{3}-10^{-7} \mathrm{~Hz}\right)$ et l'analyse enthalpique différentielle $\left(10^{0}-10^{-3} \mathrm{~Hz}\right)$.

D'autre part, le cyclohexanol est le premier cristal vitreux historiquement connu et Séki et al. ont publié l'existence de phases cristallines vitreuses dans le cyclopentanol [5] et le cycloheptanol [6]; la série des cycloalcools nous a donc paru être un bon objet d'étude.

2. Méthodes expérimentales. - 2.1 RMN LARGE BANDE. - Les expériences ont été effectuées dans le Département de Physico-Chimie du CEA-CEN Saclay sur un spectromètre JEOL, type JNM 40, $40 \mathrm{MHz}$, muni de son accessoire basse température $(110-300 \mathrm{~K})$, aimablement mis à notre disposition par Dr. C. Chachaty que nous remercions ici. Les échantillons utilisés mesuraient $50 \mathrm{~mm}$ de haut et $18 \mathrm{~mm}$ de diamètre.

2.2 Thermocourants DE DÉPOlarisation (TCD). - Cette méthode, inventée par Bucci et Fieschi [7], consiste à polariser le milieu à étudier par un champ électrique non saturant, puis à geler la configuration des molécules par refroidissement sous champ, ensuite à supprimer le champ et à réchauffer lentement afin d'enregistrer le thermocourant de dépolarisation en fonction de la température. Cette méthode, qui s'apparente aux mesures d'absorption diélectrique, permet d'obtenir des informations à de très basses fréquences, inaccessibles par les méthodes conventionnelles. Dans ce travail, nous avons utilisé la méthode mathématique d'exploitation des résultats mis au point par Heitz et Szwarc [8] qui généralise celle de Van Turnhout [9]. Les échantillons étudiés se présentent sous la forme de disques de $20 \mathrm{~mm}$ de diamètre et $2 \mathrm{~mm}$ d'épaisseur.

2.3 ANALYSE ENTHALPIQUE DIFFÉRENTIELLE (AED). - Les expériences ont été réalisées sur un analyseur enthalpique différentielle à balayage (Perkin-Elmer DSC-2C) avec son accessoire basse température (95-325 K). Les quantités d'échantillons utilisées variaient entre 3 et $15 \mathrm{mg}$.

2.4 Produits. - Les produits (cyclobutanol, cyclopentanol, cyclohexanol, cycloheptanol et cyclooctanol) provenaient de chez Aldrich avec une pureté commerciale supérieure à $99 \%$. Ils ont été généralement utilisés sans purification préalable, mais après avoir séjourné sur tamis moléculaires afin d'éviter leur hydratation. Les produits ont été systématiquement dégazés avant toute expérience en RMN.

3. Résultats expérimentaux. - 3.1 RMN LARGE BANDE. - Seul, le cyclobutanol, disponible en trop petite quantité, n'a pas été étudié. Les mesures de largeur de raie ont été effectuées en températures croissantes (après trempe de l'échantillon dans l'azote liquide) et en températures décroissantes (à partir de $200 \mathrm{~K}$ ). Dans tous les cas, la vitesse de trempe a été suffisante pour conduire au cristal vitreux.

Pour chaque composé étudié, la largeur de raie (mesurée entre les deux pics de la dérivée), dont la valeur à basse température correspond à celle du réseau rigide au sens de la RMN (environ $15 \mathrm{G}$ ), décroît dans un intervalle d'environ $100 \mathrm{~K}$ pour atteindre une faible valeur (environ $3 \mathrm{G}$ ) 
caractéristique d'une phase réorientationnelle. La figure 1 représente la largeur de raie mesurée et le second moment calculé à partir de ces mesures pour le cycloheptanol. On obtient le même type de courbe pour le cyclooctanol. En revanche, dans le cas du cyclohexanol et du cyclopentanol, la partie médiane de la courbe ne peut être parcourue : en effet, l'échantillon recristallise dans la phase stable dans ce domaine de température, aussi bien en températures croissantes que décroissantes. Eguchi et al. avaient obtenu des résultats analogues dans le cas du cyclohexanol [10].

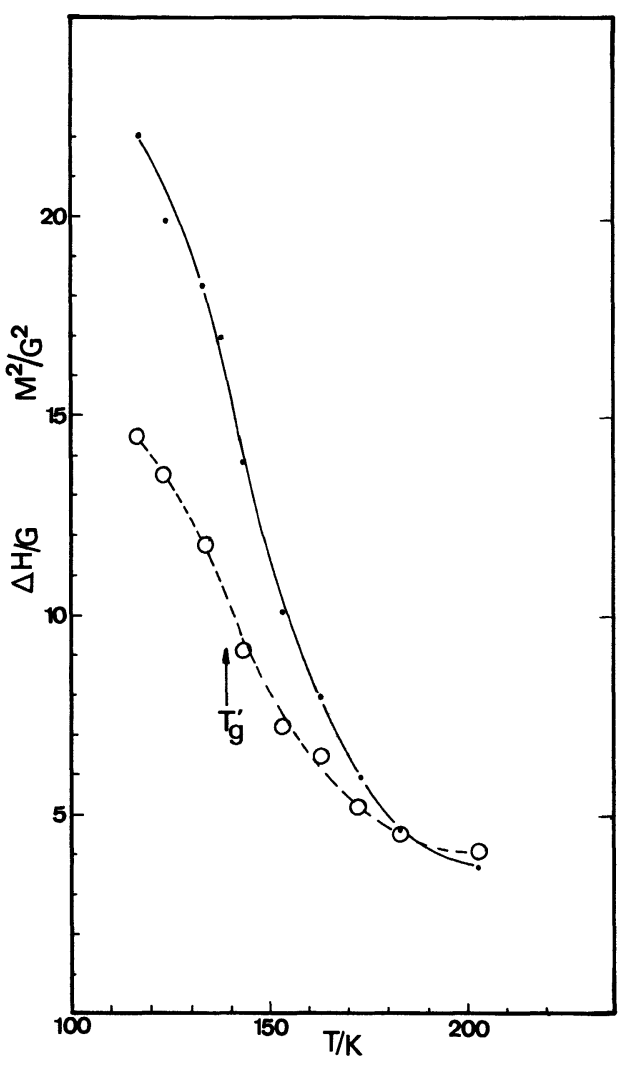

Fig. 1. - Largeur de raie RMN (O) et second moment (๑) pour le cycloheptanol trempé en fonction de la température.

[NMR line width $(O)$ and second moment $(\bullet)$ in quenched cycloheptanol as a function of temperature.]

Il faut noter que, en ce qui concerne le cyclohexanol, une purification par distillation sur tamis moléculaires, suivie d'un dégazage, permet d'éviter la recristallisation. Par ailleurs, dans ce cas, la courbe $\Delta H=f(T)$ est décalée, par rapport à celle du composé non purifié, d'environ $10 \mathrm{~K}$ vers les hautes températures. On peut rapprocher ce résultat de l'« amollissement » bien connu des verres sous l'effet de certaines impuretés.

Dans tous les cas étudiés, la température de transition $T_{\mathrm{g}}^{\prime}$, que nous proposons d'appeler « vicrale » (contraction de vitreux et de cristal), tirée d'expériences de calorimétrie (voir tableau I), se situe dans la première moitié de la décroissance de la courbe (voir Fig. 1). Un réexamen des résultats obtenus par Gutowski et Takeda [11] dans le cas du 1,2 difluoro, tétrachloroéthane (dont on sait maintenant qu'il donne un cristal vitreux [12]), révèle un comportement identique. 
On peut en inférer que ce comportement est caractéristique des cristaux vitreux. L'analogie de l'ensemble de ces résultats avec ceux obtenus dans les quelques verres étudiés de la sorte (par ex. [13, 14]), nous conforte dans cette hypothèse. Ainsi, la transition vicrale intervient à une température où la valeur du second moment est nettement inférieure à celle qui caractérise le réseau rigide; on peut donc en conclure, à l'instar d'Eguchi et al. [10], que le cristal vitreux n'est pas un « milieu mort » et qu'il est encore le siège de mouvements de réorientation de grande amplitude dont la fréquence est de l'ordre de $10^{5} \mathrm{~Hz}$ à $T_{\mathrm{g}}^{\prime}$.

Tableau I. - Propriétés thermodynamiques des cycloalcools.

[Thermodynamic properties of the cycloalcohols.]

\begin{tabular}{|c|c|c|c|c|c|c|}
\hline Composé & $\frac{T_{\mathrm{f}}}{\mathrm{K}}$ & $\frac{\Delta H_{\mathrm{f}}}{\mathrm{J} \cdot \mathrm{mole}^{-1}}$ & $\frac{T_{\mathrm{t}}}{\mathrm{K}}$ & $\frac{\Delta H_{\mathrm{t}}}{\mathrm{J} \cdot \mathrm{mole}^{-1}}$ & $\frac{T_{\mathrm{g}}^{\prime}}{\mathrm{K}}\left({ }^{6}\right)$ & $\frac{\Delta C_{p}}{\mathrm{~J} . \mathrm{mole}^{-1} \cdot \mathrm{K}^{-1}}$ \\
\hline - & - & - & -- & - & - & - \\
\hline Cyclobutanol & 228,4 & $8530\left({ }^{1}\right)$ & 225 & $?$ & 128,8 & 37 \\
\hline \multirow[t]{5}{*}{ Cyclopentanol $\left({ }^{a}\right)$} & 251,1 & 1233 & & & & \\
\hline & & & 235,2 & 61 & & \\
\hline & & & 202,0 & 3546 & & \\
\hline & & & 196,4 & 105 & & \\
\hline & & & 184,2 & 588 & 137,7 & 30 \\
\hline \multirow[t]{4}{*}{ Cyclohexanol $\left({ }^{b}\right)$} & 293,8 & 1593 & & & $148,7\left(^{4}\right)$ & 33 \\
\hline & & & & & $146,8\left(^{5}\right)$ & 10 \\
\hline & & & $243,4\left(^{2}\right)$ & 8541 & & \\
\hline & & & $263,6\left(^{3}\right)$ & $?$ & & \\
\hline \multirow[t]{2}{*}{ Cycloheptanol $\left({ }^{a, c}\right)$} & 265,8 & 1228 & & & 136,9 & 43 \\
\hline & & & 241,0 & 616 & & \\
\hline \multirow[t]{2}{*}{ Cyclooctanol } & 283,8 & 1792 & & & 141,8 & 73 \\
\hline & & & 246,5 & 1690 & 131,9 & 40 \\
\hline
\end{tabular}

( ${ }^{1}$ ) Somme des enthalpies de fusion et de transition.

( $\left.{ }^{2}\right)$ Transition III-I.

$\left({ }^{3}\right)$ Transition II-I.

$\left({ }^{4}\right)$ Refroidissement $80 \mathrm{~K} \cdot \mathrm{min}^{-1}$.

$\left({ }^{5}\right)$ Refroidissement $20 \mathrm{~K} \cdot \mathrm{min}^{-1}$.

${ }^{(6)}$ Début de la transition au réchauffement.

(a) Réf. [5].

$($ b) Réf. [2].

$\left({ }^{c}\right)$ Réf. [6].

3.2 THERMOCOURANTS DE DÉPOLARISATION. - Le cyclobutanol n'a pas été étudié pour les mêmes raisons que précédemment. En ce qui concerne le cyclopentanol, nous n'avons pas réussi à obtenir le cristal vitreux, la géométrie expérimentale ne permettant pas un refroidissement assez rapide (dans cette expérience, le refroidissement de 250 à $85 \mathrm{~K}$ prend environ $15 \mathrm{~min}$ ).

Les résultats obtenus pour les cyclo- hexanol, heptanol et octanol sont analogues. A titre d'exemple, la figure 2 représente le thermocourant de dépolarisation enregistré dans le cas du cyclohexanol. Cette figure montre qu'il existe deux domaines de relaxation diélectrique ( $\alpha$ et $\beta$ ) comme dans la plupart des verres [15]. Pour les cristaux vitreux, Séki avait déjà décrit le domaine $\beta$ 


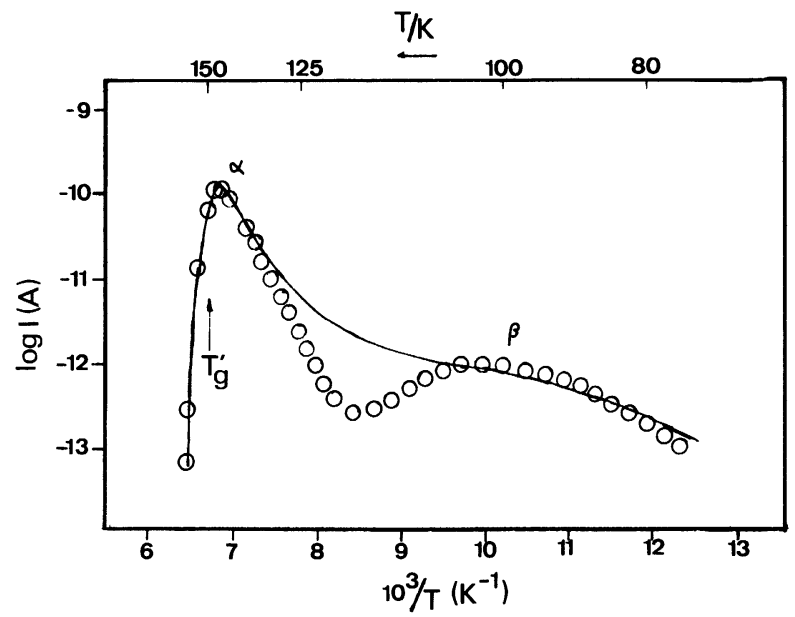

Fig. 2. - Thermocourant de dépolarisation du cyclohexanol trempé (trait plein) et courbe calculée (O).

[Thermally stimulated depolarization currents of quenched cyclohexanol (full line) and computed curve (O).]

dans le cyclohexanol [16] et Johari en avait fait état dans le néohexanol [17] $\left.{ }^{(}\right)$. La méthode mathématique d'exploitation des résultats utilisée conduit pour le domaine principal $\alpha$ (qui culmine à peu près à la température $T_{\mathrm{g}}^{\prime}+2 \mathrm{~K}$ avec une fréquence de $10^{-3} \mathrm{~Hz}$, pour une vitesse de réchauffement de 10-15 K. min $^{-1}$ ), a une distribution de temps de relaxation du type Davidson et Cole avec un paramètre de distribution $\beta$ égal à 0,5 et une énergie d'activation apparente de $\sim 60 \mathrm{~kJ} . \mathrm{mol}^{-1}$. La figure 2 montre que l'ajustement est de très bonne qualité dans un domaine d'environ $10 \mathrm{~K}$ entourant $T_{\mathrm{g}}^{\prime}$. Quant au domaine $\beta$, il correspond à une distribution de type Davidson et Cole avec un paramètre de distribution de 0,25 et une énergie d'activation apparente de $15 \mathrm{~kJ} . \mathrm{mol}^{-1}$. La figure 2 montre que, dans ce cas, l'ajustement est un peu moins bon.

L'énergie d'activation que nous avons calculée n'a d'autre prétention que de décrire la façon dont le phénomène varie en fonction de la température; elle ne fait référence à aucun modèle. De même, les propriétés diélectriques n'ont pas été décrites en termes d'une équation du type Vogel [19] - Fulcher [20] - Tammann [21] qui n'aurait de valeur - empirique - que dans un domaine de température bien supérieur à $T_{\mathrm{g}}^{\prime}$.

Dans l'image naive mais habituelle selon laquelle une transition vitreuse est liée au "gel» de certains degrés de liberté, nous nous attendions, pour la transition vicrale, au " gel » de certaines réorientations moléculaires comme semblent l'indiquer les $200 \mathrm{~s}$ du temps de relaxation révélé par les TCD à $T_{\mathrm{g}}^{\prime}$. Or, les $10^{-4} \mathrm{~s}$ de la RMN montrent, paradoxalement, qu'il n'en est rien localement.

3. 3 ANALYSE ENTHALPIQUE DIFFÉRENTIELLE. - Les 5 cycloalcools ont pu être étudiés par AED et les comportements déjà publiés par ailleurs pour 3 d'entre eux $[2,5,6]$ ont en général été retrouvés et/ou précisés. Les résultats détaillés seront publiés par ailleurs.

La phase mère, dont la nature cristalline et non liquide a été systématiquement vérifiée, a été refroidie (de $320 \mathrm{~K} \cdot \mathrm{min}^{-1}$ à $20 \mathrm{~K} \cdot \mathrm{min}^{-1}$ selon le domaine de température pertinent) puis réchauffée à $10 \mathrm{~K} \cdot \mathrm{min}^{-1}$.

( $\left.{ }^{1}\right)$ Dans ce dernier cas toutefois, les mesures calorimétriques portant sur des échantillons de l'ordre de $10 \mathrm{mg}$, refroidis à partir du liquide, n'ont jamais conduit au cristal vitreux mais toujours au verre [18]. 
La transition vicrale a été observée dans tous ces composés, ainsi que le montre le tableau I. Il semble que l'importance de l'incrément de chaleur spécifique à $T_{\mathrm{g}}^{\prime}$ soit corrélée à l'enthalpie de la transition évitée, lorsque celle-ci peut être clairement attribuée, qui n'est d'ailleurs pas nécessairement celle dont l'enthalpie est la plus grande.

Par ailleurs, et suivant en celà le comportement habituel des verres, $T_{\mathrm{g}}^{\prime}$ se situe à une température d'autant plus haute que la vitesse de refroidissement a été plus grande (variation de $\sim 2 \mathrm{~K}$ pour des vitesses de trempe variant de 80 à $20 \mathrm{~K} \cdot \mathrm{min}^{-1}$ ).

La figure 3, qui présente les résultats obtenus pour le cyclooctanol, montre qu'il existe une transition vicrale dans la phase cristalline II. Ce comportement laisse penser qu'il devrait exister une autre phase plus stable à basse température. Ainsi donc, il y aurait dans le cyclooctanol deux phases cristal vitreux différentes comme il en est dans le cyclohexène [22].



Fig. 3. - Thermogramme AED du cyclooctanol : — Après refroidissement lent ; - - Après trempe. [DSC analysis of cyclooctanol : —_ After slow cooling ; - - After quenching.]

4. Discussion et conclusions. - Les vitesses de réchauffement en AED et en TCD sont pratiquement les mêmes et, dans les deux cas, la fréquence au maximum de l'effet enregistré est de l'ordre de $10^{-3} \mathrm{~Hz}$. On peut donc penser à une origine commune des phénomènes révélés par les deux techniques : relaxation diélectrique $\alpha$ et accident de chaleur spécifique. Si l'on rapproche ce fait de l'autre constat expérimental qui ressort des mesures RMN, selon lequel des mouvements de réorientations moléculaires à une fréquence de $10^{5} \mathrm{~Hz}$ persistent à des températures inférieures à $T_{\mathrm{g}}^{\prime}$, on est conduit à écarter l'hypothèse, pourtant la plus simple, d'un gel des sauts réorientationnels lors du passage de la transition vicrale. De la même façon, il faut rappeler que Séki et al. [2], comparant des résultats obtenus par calorimétrie et spectroscopie IR dans le cas du cyclohexanol cristal vitreux, n'avaient pu dégager une corrélation simple entre des modes inter- ou intramoléculaires et d'éventuels degrés de liberté gelés.

Par ailleurs, il faut remarquer que, pour ce même composé, Eguchi et al. [10] ont, par des mesures de temps de relaxation spin-réseau RMN, trouvé, pour la réorientation moléculaire, une énergie d'activation apparente qui passe de $8 \mathrm{~kJ} \cdot \mathrm{mol}^{-1}$, dans le cristal vitreux à $80 \mathrm{~K}$, à $13 \mathrm{~kJ} . \mathrm{mol}^{-1}$ vers $170 \mathrm{~K}$ dans la phase plastique métastable. Ces valeurs sont à rapprocher de 
l'énergie d'activation apparente $\left(15 \mathrm{~kJ} \mathrm{~mol}^{-1}\right)$ mesurée ici par TCD pour le domaine $\beta$ dans tous lès composés étudiés. On est donc en droit de se demander si la variation de la largeur de raie observée en RMN aux alentours de $T_{\mathrm{g}}^{\prime}$ ne correspond pas plutôt aux phénomènes observés à plus basse température, mais à une autre échelle de temps et qui se trouvent à l'origine de la relaxation $\beta$, à certaines réorientations moléculaires par exemple.

Pour les verres moléculaires, le comportement de la viscosité dans la région autour de $T_{\mathrm{g}}$ laisse penser que les mouvements de translation se bloquent à cette température. Il s'ensuit que la relaxation $\beta$, à plus basse température, ne pourrait affecter que des modes réorientationnels. Arndt et Jonas [23] font d'ailleurs cette hypothèse dans leur étude du verre d'isopropylbenzène. Or, dans le cas des cristaux vitreux moléculaires, les mouvements de translation sont déjà bloqués depuis la solidification, et, si la relaxation $\beta$ et le rétrécissement de la raie $\mathrm{RMN}$ ont effectivement la même origine, seuls les mouvements de réorientations peuvent être invoqués pour expliquer aussi bien la relaxation $\alpha$ que la relaxation $\beta$. Vu l'extrême similitude de comportement des verres moléculaires et des cristaux vitreux, la conclusion s'impose que les phénomènes moléculaires qui président aux différentes transitions, vitreuses ou vicrales, sont composites et mettent probablement en jeu un ensemble de modes moléculaires, mais à des échelles d'espace [24] et de temps différentes et à divers degrés de coopérativité.

C'est la raison pour laquelle nous nous proposons d'étendre nos investigations au domaine des fréquences qui va de $3 \times 10^{7}$ à $10^{12} \mathrm{~Hz}$.

\section{Bibliographie}

[1] Westrum Jr., D. F., et McCullough, J. P., in Physics and Chemistry of the Organic Solid State, Ed. D. Fox, M. M. Labes and A. Weissberger, Vol. 1 (Interscience, New York) 1963, p. 1-178.

[2] Adachi, K., Suga, H. et SÉKi, S., Bull. Chem. Soc. Japan 41 (1968) 1073.

[3] Otsûbo, A. et Sugawara, T., Sci. Rep. Res. Inst. Tohoku Univ. A 7 (1955) 583.

[4] Ceccaldi, D., Dénoyer, F., Lambert, M. et Szwarc, H., J. Physique Lett. 41 (1980) L-365.

[5] Adachi, K., Suga, H. et SéKi, S., Bull. Chem. Soc. Japan 43 (1970) 1916.

[6] 'AdACHI, K., SUga, H. et SéKI, S., Bull. Chem. Soc. Japan 45 (1972) 1960.

[7] BuCCI, C. et FiesCHI, R., Phys. Rev. Lett. 12 (1964) 16; Phys. Rev. 148 (1966) 816.

[8] Heitz, R. J. et Szwarc, H., Revue Phys. Appl. 15 (1980) 687.

[9] Van Turnhout, J., Central Lab. TNO delft Commun. no 471 (1972).

[10] Eguchi, T., Soda, G. et ChiHaRA, H., J. Magn. Res. 23 (1976) 55.

[11] Gutowski, H. S. et Takeda, M., J. Phys. Chem. 61 (1957) 95.

[12] Kishimoto, K., Suga, H. et SéKi, S., Bull. Chem. Soc. Japan 51 (1978) 1691.

[13] McCall, D., Acc. Chem. Rev. 4 (1971) 223.

[14] Ellis, B. et McDonald, M. P., J. Non-Cryst. Solids 1 (1969) 166.

[15] Johari, G. P. et Goldstein, M., J. Chem. Phys. 53 (1970) 2372.

[16] Adachi, K., Suga, H., Séki, S., Kubota, S., Yamaguchi, S., Yano, O. et Wada, Y., Mol. Cryst. Liq. Cryst. 18 (1972) 345.

[17] Johari, G. P., in The Glass Transition and the Nature of the Glassy State, Ed. M. Goldstein et R. Simha, Ann. New York Acad. Sci. 279 (1976) 117.

[18] Dworkin, A., in 69th Faraday Discussion, after Paper no 20, Exeter (UK), (1980).

[19] Vogel, H., Physik Z. 22 (1921) 645.

[20] FulCher, G. S., J. Am. Ceram. Soc. 8 (1925) 339.

[21] Tammann, A., Z. Anorg. Allgem. Chem. 156 (1926) 245.

[22] Haida, O., Suga, H. et Séki, S., Bull. Chem. Soc. Japan 50 (1977) 802.

[23] Arndt, E. et Jonas, J., J. Phys. Chem. 85 (1981) 463.

[24] Johari, G. P., Philos. Mag. 41 (1980) 41. 